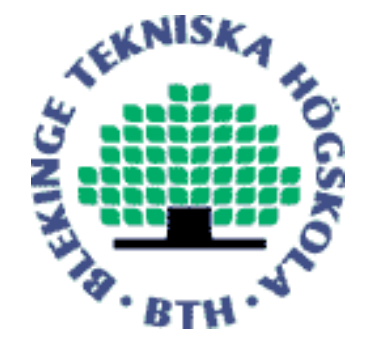

Copyright (C) 2014 IEEE.

Citation for the published paper:

Low-complexity codebook-based beamforming with four transmit antennas and quantized feedback channel

Samuel Tumelero Valduga, Dimas Irion Alves, Renato Machado, Andrei Piccinini Legg, Murilo Bellezoni Loiola

Wireless Communications and Networking Conference (WCNC)

2014 Istanbul

This material is posted here with permission of the IEEE. Such permission of the IEEE does not in any way imply IEEE endorsement of any of BTH's products or services Internal or personal use of this material is permitted. However, permission to reprint/republish this material for advertising or promotional purposes or for creating new collective works for resale or redistribution must be obtained from the IEEE by sending a blank email message to pubs-permissions@iee.org.

By choosing to view this document, you agree to all provisions of the copyright laws protecting it. 


\title{
Low-Complexity Codebook-Based Beamforming with Four Transmit Antennas and Quantized Feedback Channel
}

\author{
Samuel T. Valduga*, Dimas I. Alves ${ }^{\dagger}$, Renato Machado*‡, Andrei P. Legg*, and Murilo B. Loiola ${ }^{\S}$ \\ * Federal University of Santa Maria - UFSM \\ Santa Maria, RS, 97105-900, Brazil \\ $\dagger$ Telecommunications Research and Development Center - CPqD \\ Campinas, SP, 13086-902, Brazil \\ $\ddagger$ Blekinge Institute of Technology - BTH \\ Karlskrona, SE-371 79, Sweden \\ $\S$ Universidade Federal do ABC - UFABC \\ Santo Andr, SP, 09210-170, Brazil \\ E-mail: samucatv@gmail.com, dalves@cpqd.com.br, renatomachado@ieee.org, andrei.legg@gmail.com, murilo.loiola@ufabc.edu.br
}

\begin{abstract}
In this paper we propose a low-complexity codebook-based beamforming with four transmit antennas and quantized feedback channel. The codebook design aggregates the effect of power allocation and phase rotation through a simple quantized transmit scheme. The codebook-based beamforming uses the feedback information in order to maximize the instantaneous signal-to-noise ratio (SNR) at the receiver. As a result, the proposed scheme presents an array gain. An SNR analysis is performed and it is used to find the optimal feedback information in the sense of maximizing the instantaneous SNR. A bit error rate (BER) analysis for a quantized feedback channel is also derived and it is used to compare to the results obtained for the proposed scheme under different levels of quantization. Simulations are performed over quasi-static flat Rayleigh fading channels for different closed-loop codebook-based schemes with four transmit antennas and unitary transmission rate. Results illustrate that the proposed scheme achieves full diversity order and outperforms other good schemes in terms of array gain.
\end{abstract}

\section{INTRODUCTION}

Quantized transmit beamforming is an interesting realworld solution for multiple-input multiple-output (MIMO) systems since this directional signal can improve the signalto-noise ratio (SNR) at the receiver enhancing the channel capacity [1] and reducing the symbol or bit error probability of the system [2]. These improvements, obviously, depend on the the amount of feedback available. When full channel state information (CSI) is available to the transmitter, optimal beamforming is the best strategy to be considered [3]. In fact, transmit beamforming uses all the transmit antennas and distributes the total average power non-uniformly among them.

Deployment of multiple antennas is normally associated with high cost since multiple antennas usually require an equal number of radio frequency (RF) chains (transmit amplifiers, modulators, etc.), which are expensive and power-consuming. To circumvent this problem, a subset of the available antennas can be selected, thereby reducing the number of RF chains. The amount of feedback required in this case is much smaller.
Such approach is called transmit antenna selection (TAS). The most well-known TAS scheme has been proposed by Gore and Paulraj [4] and later, an extension of this idea was presented in [5].

Another efficient way of using the feedback bits is through a codebook-based (CBB) design, where the feedback information is used to select a codeword from a finite codebook [2]. Along the last years, several works have investigated the use of quantized feedback in multiple-antenna CBB systems [6]-[12], which is currently considered in the LTE standard [13].

In [12], an analysis on symbol error rate of maximum ratio transmission, transmit antenna selection, and codebookbased beamforming in correlated Rayleigh fading channels was presented. Zhu et al. have derived a universal upper bound on the average SER of those three schemes for a quantized feedback information.

In [10], a class of extended orthogonal space-time block codes (EO-STBCs) for MIMO channels considering four transmit antennas over quasi-static flat fading channels was proposed. The transmission rate of this scheme is one, since two symbols are transmitted over two symbol periods. In this work, phase information is fed back from the receiver to the transmitter and, based on this information, phases of certain symbols are rotated. As a result, the EO-STBC presented in [10] outperformed previous rate-one closed-loop STBCs with four transmit antennas.

In [11], Choi et al. have proposed an interesting phasefeedback-assisted scheme with four transmit antennas which uses a preprocessor for combining two Alamouti codes in terms of Frobenius norm maximization. They have shown that full diversity is achieved by the combining effect at the preprocessor. Also the phase feedback is utilized to increase the coding gain. Herein, we refer to this scheme as Alamouticode based scheme (ACBS). 


\section{A. Contribution and Objectives}

By knowing that the feedback rate is a critical aspect in real-world wireless systems, we propose a low-complexity codebook-based beamforming with four transmit antennas and quantized feedback channel. The feedback index (phases) is used to maximize the instantaneous signal-to-noise ratio (SNR). The preprocessing considered for the proposed scheme is based on the designs presented in [10] and [11].

We also addressed an SNR analysis which is considered to find the optimal (non-quantized) feedback phases in terms of instantaneous signal-to-noise ratio maximization. An upper bound on the average BER performance is obtained, which is based on the analysis presented in [12] and [14]. Another important feature of the proposed scheme is the signal detection which is based on linear processing, leading to a lowcomplexity receiver.

A quantized feedback analysis of the proposed scheme over quasi-static flat Rayleigh fading channels is also performed. Simulation results illustrate that the proposed scheme outperforms other good phase feedback codebook-based schemes with four transmit antennas.

\section{B. Organization and Some Notations}

The rest of the paper is organized as follows. Section II presents the system model. Section III addresses the lowcomplexity codebook-based beamforming with four transmit antennas and quantized feedback channel. Furthermore an SNR analysis for the proposed scheme is provided. In Section IV, a BER analysis for a quantized feedback channel is derived and used later in Section V, where simulations are taken into account. Finally, Section VI presents some concluding remarks.

Throughout this paper, normal letters represent scalar quantities, boldface lowercase letters indicate vectors, and boldface uppercase letters indicate matrices. The superscripts ' $T$ ' and ' $*$ ' represent the transpose and the complex conjugate operation, $|\cdot|^{2}$ denotes the modulus squared of a complex number and $\Re\{\cdot\}$ the real part of a complex number.

\section{SYSTEM MODEL}

Consider a MIMO system with $M_{T}=4$ transmit antennas at the transmitter and $M_{R}$ receive antennas at the receiver. The channel is assumed to be quasi-static, flat Rayleigh fading so that the channel is constant over a frame ( $\tau$ symbol periods) and vary randomly from one frame to the next. The transmission model consists of linear processing, as described in [11]. With some slight modifications, we arrive at

$$
\mathbf{Y}=\mathbf{P H I}_{S N R}+\mathbf{N},
$$

where $\mathbf{Y}$ is the $\tau \times M_{R}$ matrix of the received signals and $\mathbf{P}$ is the $\tau \times M_{T}$ matrix of processed transmitted signals with unit average energy. Let $\mathcal{C N}(0, \mathbf{R})$ represent the joint p.d.f. (probability density function) of a zero-mean circularly symmetric complex normal random vector with covariance matrix $\mathbf{R}$. Then, $\mathbf{N}$ is the $\tau M_{R}$ matrix $\mathcal{C N}\left(\mathbf{0}, \mathbf{I}_{\tau M_{R}}\right)$ representing the joint p.d.f. of the i.i.d. (independent and identically distributed) additive Gaussian noise samples with unit variance, $\mathbf{H}$ is the $M_{T} M_{R}$ MIMO channel characterized by the p.d.f.
$\mathcal{C N}\left(\mathbf{0}, \mathbf{I}_{M_{T} M_{R}}\right)$, and $\mathbf{I}_{S N R}$ is the $M_{R} \times M_{R}$ diagonal matrix, where the $i$-th diagonal element of this matrix is given by $\sqrt{\rho}$, with $\rho=E_{b} / N_{0}$, which means the SNR in each receive antenna, $E_{b}$ is the average transmitted bit power, and $N_{0}$ is the noise variance.

We assume that the information bits are mapped into a base-band unitary average energy constellation, such as PSK or QAM constellations, given rise to $Q$ data symbols $\left\{s_{q}\right\}$, $q=1, \ldots, Q$, to be transmitted over $\tau$ symbol periods. In this paper, we consider only schemes with unitary spatial transmission rate $(R=Q / \tau=1)$. We also assume that the channel coefficients are perfectly estimated at the receiver and that there is a reliable feedback channel through which $b$ bits can be sent to the transmitter.

\section{The Proposed SCHEME}

We start this section by considering how the transmission of the proposed scheme is performed. After, the linear receiver for $M_{R}$ receive antennas will be addressed.

\section{A. The Transmitter}

The proposed scheme has four transmit antennas, where only one information symbol, $s$, is transmitted per transmit period. The symbol $s$ is preprocessed by a complex transmission codeword $\mathbf{x}$ :

$$
\mathbf{p}=\mathbf{x} s
$$

where

$$
\begin{aligned}
\mathbf{x} & =\left[\mathbf{v}_{\varphi}\left(\left[\begin{array}{cc}
\cos (\theta) / \sqrt{2} & 0 \\
0 & \sin (\theta) / \sqrt{2}
\end{array}\right] \otimes \mathbf{I}_{2}\right)\right], \\
& =\frac{1}{\sqrt{2}}\left[\begin{array}{l}
\cos (\theta) e^{j \varphi_{0}} \\
\cos (\theta) e^{j \varphi_{1}} \\
\sin (\theta) e^{j \varphi_{0}} \\
\sin (\theta) e^{j \varphi_{2}}
\end{array}\right],
\end{aligned}
$$

where $\otimes$ is the kronecker product, $\varphi_{i}, i=0,1,2$, represents a phase, $\mathbf{I}_{2}$ is the 2-by-2 identity matrix, and

$$
\mathbf{v}_{\varphi}=\left[\begin{array}{llll}
e^{j \varphi_{0}} & e^{j \varphi_{1}} & e^{j \varphi_{0}} & e^{j \varphi_{2}}
\end{array}\right] .
$$

The factor $\frac{1}{\sqrt{2}}$ is used for normalizing the transmit power.

For simplicity of analysis, we assume that $\varphi_{0}=0$. Henceforth we follow with this simplification since $\varphi_{0}$ is used as a reference phase only.

Before that each transmission takes place, the receiver feeds back $b$ bits to the transmitter and this information is used appropriately by the preprocessor for choosing the codeword

$$
\mathbf{x}_{\max } \in \mathbf{X}=\left[\mathbf{x}_{1}, \mathbf{x}_{2}, \ldots, \mathbf{x}_{N}\right],
$$

where $N=2^{b} . \mathbf{x}_{\max }$ is the codeword that maximizes the instantaneous SNR. As we can observe, the codebook is a function of $\varphi_{1}, \varphi_{2}$ and $\theta$. Therefore, the codebook is defined based on the sets adopted for those variables.

Next, we present the receiver for $M_{R}$ receive antennas, the SNR analysis, which is used to find the optimal values for $\varphi_{1}$, $\varphi_{2}$ and $\theta$, and also how the proposed scheme is performed with a quantized feedback. 


\section{B. Linear Receiver for $M_{R}$ receive antennas}

From (1) and (2), the received signal $\mathbf{y}$ for $M_{R}$ receive antennas can be rewritten as

$$
\mathbf{y}=s \mathbf{h}_{\mathbf{p}}+\mathbf{n},
$$

where

$$
\begin{aligned}
\mathbf{h}_{\mathbf{p}} & =\mathbf{x H} \\
& =\left[h_{p 1} \ldots h_{p i} \ldots h_{p M_{R}}\right]
\end{aligned}
$$

in which

$$
\begin{aligned}
h_{p i}=\cos (\theta) h_{1, i}+\cos (\theta) e^{\varphi_{1}} h_{2, i} \\
\quad+\sin (\theta) h_{3, i}+\sin (\theta) e^{\varphi_{2}} h_{4, i}
\end{aligned}
$$

and

$$
\mathbf{H}=\left[\begin{array}{cccc}
h_{1,1} & h_{2,1} & h_{3,1} & h_{4,1} \\
\vdots & \vdots & \vdots & \vdots \\
h_{1, M_{R}} & h_{2, M_{R}} & h_{3, M_{R}} & h_{4, M_{R}}
\end{array}\right]^{\mathrm{T}}
$$

where $\mathbf{n}$ is the $1 \times M_{R}(\tau=1)$ additive white Gaussian noise vector, and $h_{i, j}$ denotes the path gain from the $i$-th transmit antenna to the $j$-th receive antenna.

Considering the received signal in (3) and employing the maximal ratio combining (MRC) technique, the following linear processing produces the desired inputs to the maximumlikelihood detection:

$$
\begin{aligned}
& \tilde{s}= y_{1} h_{p 1}^{*}+\ldots+y_{i} h_{p i}^{*}+\ldots+y_{M_{R}} h_{p M_{R}}^{*} \\
&=s\left[\left(\sum_{i=1}^{M_{R}}\left(\left|h_{1, i}\right|^{2}+\left|h_{2, i}\right|^{2}\right)+\beta_{c}\right) \cos (\theta)^{2}\right. \\
&+\left(\sum_{i=1}^{M_{R}}\left(\left|h_{3, i}\right|^{2}+\left|h_{4, i}\right|^{2}\right)+\beta_{s}\right) \sin (\theta)^{2} \\
&\left.+\beta_{c s} \cos (\theta) \sin (\theta)\right] \sqrt{\rho} / 2+\sum_{i=1}^{M_{R}} h_{p i}^{*} n_{i}
\end{aligned}
$$

where $\eta_{i}$ is the i.i.d. additive Gaussian noise samples with unit variance, $\beta_{c}=\sum_{i=1}^{M_{R}} \beta_{c i}, \beta_{s}=\sum_{i=1}^{M_{R}} \beta_{s i}, \beta_{c s}=\sum_{i=1}^{M_{R}} \beta_{c s i}$ and

$$
\begin{aligned}
\beta_{c i} & =2 \Re\left\{h_{1, i} h_{2, i}^{*} e^{-j \varphi_{1}}\right\} \\
\beta_{s i} & =2 \Re\left\{h_{3, i} h_{4, i}^{*} e^{-j \varphi_{2}}\right\} \\
\beta_{c s i} & =2 \Re\left\{\left(h_{1, i}+h_{2, i} e^{j \varphi_{1}}\right)\left(h_{3, i}+h_{4, i} e^{j \varphi_{2}}\right)^{*}\right\}
\end{aligned}
$$

\section{Instantaneous SNR Analysis}

For the signal obtained in (5), the instantaneous output SNR is given by

$$
\gamma=\left(\frac{g_{c} \cos ^{2}(\theta)+g_{s} \sin ^{2}(\theta)+\beta_{c s} \cos (\theta) \sin (\theta)}{2}\right) \rho
$$

where

$$
\begin{aligned}
g_{c}= & {\left[\sum_{i=1}^{M_{R}}\left|h_{1, i}\right|^{2}+\left|h_{2, i}\right|^{2}+2 \Re\left\{h_{1, i} h_{2, i}^{*} e^{-j \varphi_{1}}\right\}\right] \cos ^{2}(\theta) } \\
g_{s}= & {\left[\sum_{i=1}^{M_{R}}\left|h_{3, i}\right|^{2}+\left|h_{4, i}\right|^{2}+2 \Re\left\{h_{3, i} h_{4, i}^{*} e^{-j \varphi_{2}}\right\}\right] \sin ^{2}(\theta) } \\
& \beta_{c s}=\sum_{i=1}^{M_{R}} 2 \Re\left\{\left(h_{1, i}+h_{2, i} e^{j \varphi_{1}}\right)\left(h_{3, i}+h_{4, i} e^{j \varphi_{2}}\right)^{*}\right\}
\end{aligned}
$$

Now, we can determine the optimal $\varphi_{1}, \varphi_{2}$, and $\theta$ by differentiation of (6), yielding a maximum signal-to-noise ratio and diversity order $4 M_{R}\left(M_{T}=4\right)$. Nevertheless, it is important to mention that full diversity can also be achieved with quantized feedback (as described latter in this paper).

From (6), we observe that there is a dependence among the variables present in this optimization process, making the instantaneous SNR maximization problem hard to solve. Thus, we chose to solve this optimization problem in two steps.

First, we differentiate $\beta_{c}$ and $\beta_{s}$ with respect to $\varphi_{1}$ and $\varphi_{2}$, respectively, and set them both equal to zero. We can easily verify that those terms are maximized when

$$
\begin{aligned}
& \varphi_{1}=\arctan \left(\sum_{i=1}^{M_{R}} \frac{\sin \left(\xi_{1, i}-\xi_{2, i}\right) \alpha_{1, i} \alpha_{2, i}}{\cos \left(\xi_{1, i}-\xi_{2, i}\right) \alpha_{1, i} \alpha_{2, i}}\right) \\
& \varphi_{2}=\arctan \left(\sum_{i=1}^{M_{R}} \frac{\sin \left(\xi_{3, i}-\xi_{4, i}\right) \alpha_{3, i} \alpha_{4, i}}{\cos \left(\xi_{3, i}-\xi_{4, i}\right) \alpha_{3, i} \alpha_{4, i}}\right)
\end{aligned}
$$

where, $h_{a, b}=\alpha_{a, b} \exp \left\{\xi_{a, b}\right\}$.

Second, we differentiate (6) with respect to $\theta$. The first and second derivatives are given by

$$
\gamma^{\prime}=2 \kappa(\cos (\theta) \sin (\theta))+\beta_{c s}\left(\cos ^{2}(\theta)-\sin ^{2}(\theta)\right)
$$

and

$$
\gamma^{\prime \prime}=2 \kappa\left(\cos ^{2}(\theta)-\sin ^{2}(\theta)\right)-4 \beta_{c s}(\cos (\theta) \sin (\theta)),
$$

respectively.

Solving (9) and (10) under the conditions $\gamma^{\prime}=0$ and $\gamma^{\prime \prime}<$ 0 , we obtain the following optimal theta phase

$$
\theta_{\text {opt }}=\arctan \left(\frac{\kappa+\sqrt{\kappa^{2}+2 \beta_{c s}^{2}}}{\beta_{c s}}\right),
$$

with $\kappa=g_{s}-g_{c}$.

We decide to omit the term $\rho$ in (9) and (10), since this does not alter the final result.

\section{Quantized Feedback}

In this section, we present how to perform the proposed scheme with quantized feedback. We assume two uniform finite phase sets: $\varphi_{1 q}$ and $\varphi_{2 q} \in[0 \pi]$ and $\theta_{q} \in[-\pi / 2 \pi / 2]$. Since the receiver needs to feed back the state information about three phases, the transmitter receive, at least, three feedback bits for pre-processing the information properly (see Table I). The receiver computes and compares $2^{b-2}$ instantaneous SNR and send $b$ feedback bits to the transmitter.

Table I defines the criteria used by the receiver for feeding back the quantized vector. Each vector (or group of phases), presented in each row of Table I, defines a codeword $\mathbf{x}\left(\varphi_{1}, \varphi_{2}, \theta\right)$. Phases $\varphi_{1}$ and $\varphi_{2}$ are used to ensure that the terms $\beta_{c}$ and $\beta_{s}$ are positive numbers. As the number of feedback bits is increased, $\beta_{c}$ and $\beta_{s}$ converge to the ideal (non-quantized) solution. The third phase, i.e. $\theta$, is used to maximize the sum of the terms that compose the instantaneous SNR.

In Table I, we see how the codebooks, $\mathbf{X}_{8}$ and $\mathbf{X}_{16}$, are designed for $b=3$ and 4 feedback bits, respectively. The symbol $\sharp$ specifies the "do not care state". 
TABLE I. QUANTIZED FEEDBACK: CODEBOoK DESIGN.

\begin{tabular}{|c|c|c|c|c|c|c|}
\hline Feedback bits & $\beta_{c}$ & $\beta_{s}$ & $\beta_{c s}$ & $\kappa$ & $\theta_{q}$ & $\varphi_{1 q} / \varphi_{2 q}$ \\
\hline \multirow{8}{*}{$b=3$ bits } & $>0$ & $>0$ & $>0$ & $\sharp$ & $\frac{\pi}{4}$ & $0 / 0$ \\
\hline & $>0$ & $<0$ & $>0$ & \# & $\frac{\pi}{4}$ & $0 / \pi$ \\
\hline & $<0$ & $>0$ & $>0$ & \# & $\frac{\pi}{4}$ & $\pi / 0$ \\
\hline & $<0$ & $<0$ & $>0$ & \# & $\frac{\pi}{4}$ & $\pi / \pi$ \\
\hline & $>0$ & $>0$ & $<0$ & $\sharp$ & $\frac{-\pi}{4}$ & $0 / 0$ \\
\hline & $>0$ & $<0$ & $<0$ & $\sharp$ & $\frac{-\pi}{4}$ & $0 / \pi$ \\
\hline & $<0$ & $>0$ & $<0$ & $\sharp$ & $\frac{-\pi}{4}$ & $\pi / 0$ \\
\hline & $<0$ & $<0$ & $<0$ & $\sharp$ & $\frac{-\pi}{4}$ & $\pi / \pi$ \\
\hline \multirow{16}{*}{$b=4$ bits } & $>0$ & $>0$ & $>0$ & $>0$ & $\frac{3 \pi}{8}$ & $0 / 0$ \\
\hline & $>0$ & $<0$ & $>0$ & $>0$ & $\frac{5 \pi}{8}$ & $0 / \pi$ \\
\hline & $<0$ & $>0$ & $>0$ & $>0$ & $\frac{3 \pi}{8}$ & $\pi / 0$ \\
\hline & $<0$ & $<0$ & $>0$ & $>0$ & $\frac{3 \pi}{8}$ & $\pi / \pi$ \\
\hline & $>0$ & $>0$ & $>0$ & $<0$ & $\frac{\pi}{8}$ & $0 / 0$ \\
\hline & $>0$ & $<0$ & $>0$ & $<0$ & $\frac{\pi}{8}$ & $0 / \pi$ \\
\hline & $<0$ & $>0$ & $>0$ & $<0$ & $\frac{\pi}{8}$ & $\pi / 0$ \\
\hline & $<0$ & $<0$ & $>0$ & $<0$ & $\frac{\pi}{8}$ & $\pi / \pi$ \\
\hline & $>0$ & $>0$ & $<0$ & $>0$ & $\frac{-\pi}{8}$ & $0 / 0$ \\
\hline & $>0$ & $<0$ & $<0$ & $>0$ & $\frac{-\pi}{8}$ & $0 / \pi$ \\
\hline & $<0$ & $>0$ & $<0$ & $>0$ & $\frac{-\pi}{8}$ & $\pi / 0$ \\
\hline & $<0$ & $<0$ & $<0$ & $>0$ & $\frac{-\pi}{8}$ & $\pi / \pi$ \\
\hline & $>0$ & $>0$ & $<0$ & $<0$ & $\frac{-3 \pi}{8}$ & $0 / 0$ \\
\hline & $>0$ & $<0$ & $<0$ & $<0$ & $\frac{-3 \pi}{8}$ & $0 / \pi$ \\
\hline & $<0$ & $>0$ & $<0$ & $<0$ & $\frac{-3 \pi}{8}$ & $\pi / 0$ \\
\hline & $<0$ & $<0$ & $<0$ & $<0$ & $\frac{-3 \pi}{8}$ & $\pi / \pi$ \\
\hline
\end{tabular}

\section{BER ANALYSIS}

In this section, we present a BER analysis for a codebookbased beamforming. This analysis is performed for PSK constellations.

The BER of $M$-ary PSK constellation conditioned on the instantaneous SNR $\gamma$ can be given by [15]:

$$
\operatorname{BER}(\gamma)=\frac{1}{\pi} \int_{0}^{\frac{(M-1) \pi}{M}} \exp \left(-\frac{C \gamma}{\sin ^{2}(\theta)}\right) d \theta
$$

where $M$ is the constellation size and $C=\sin ^{2}(\pi / M)$.

Taking the expectation over (12), the average BER can be written as

$$
\overline{B E R}(\gamma)=\mathbb{E}_{h}\{B E R(\gamma)\},
$$

where $\mathbb{E}_{h}$ is the expectation operator with respect to $h$.

Now, by assuming that the transmitter uses feedback information for choosing a codeword $\mathbf{x}$, which belongs to a certain finite codebook $\mathbf{X}$, such that the instantaneous SNR is maximized, we can rewritten (13) as

$$
\overline{\operatorname{BER}}(\rho)=\frac{1}{\pi} \int_{0}^{\frac{(M-1) \pi}{M}} \mathbb{M}\left(-\frac{C \rho}{\sin ^{2}(\theta)} ; \mathbf{X}\right) d \theta
$$

where $\mathbb{M}\left(-\frac{C \rho_{x}}{\sin ^{2}(\theta)} ; \mathbf{X}\right)$ denotes the value of the function

$$
\begin{aligned}
\mathbb{M}(t ; \mathbf{X}) & \triangleq \mathbb{E} \exp \left(-t \max _{\mathbf{x} \in \mathbf{X}}\left|\mathbf{h}_{\mathbf{p}}\right|^{2}\right), t \geq 0 \\
& =\mathbb{E} \exp \left(-t \max _{\mathbf{x} \in \mathbf{X}}|\mathbf{x} \mathbf{H}|^{2}\right), t \geq 0
\end{aligned}
$$
$(15)$ :

As in [12], we consider the following approximation of

$$
\beta(\mathbf{X})=\lim _{t \rightarrow \infty} t^{M_{T} M_{R}} \mathbb{M}(t ; \mathbf{X})
$$

in which the values assumed by $\beta(t, \mathbf{X})$ depend on the set of codewords in $\mathbf{X}$.
This asymptotic approximation is fundamental for our analysis since an exact expression is very hard to obtain. However, if the codebook is well designed, and that is our case, then the Voronoi cells of the codewords can be approximated by 'spherical caps' on the surface of hypersphere, which simplifies considerably the BER analysis [14].

Based on the results presented in [14] and [12] we have the following approximation of (16)

$$
\begin{array}{r}
\beta(\mathbf{X})=C_{1}\left(M_{T} M_{R}-1\right) !\left(M_{T}-1\right) N \\
\times \sum_{n=0}^{M_{T}-2}\left(\begin{array}{c}
M_{T}-2 \\
n
\end{array}\right) \frac{(-1)^{n}\left(C_{2}^{-\left(M_{T} M_{R}-1-n\right)}-1\right)}{M_{T} M_{R}-1-n}
\end{array}
$$

where

$$
C_{1}=M_{T} M_{R} \prod_{n=1}^{\min \left(M_{T} M_{R}\right)} \frac{\left(\min \left(M_{T} M_{R}\right)-n\right) !}{\left(M_{T} M_{R}-1\right) !}
$$

and

$$
C_{2}=1-N^{\frac{-1}{M_{T}-1}}
$$

\section{A. An upper bound on the BER performance}

In this section, we continue with the analysis of $\beta(\mathbf{X})$, whose result will be used in (14) culminating in an upper bound on the average BER.

The normalized channel is defined by $\tilde{\mathbf{h}}_{p}=\frac{\mathbf{h}_{\mathbf{p}}}{\left|\mathbf{h}_{\mathbf{p}}\right|}$ where $\left|\mathbf{h}_{\mathbf{p}}\right|$ is independent of its direction. Furthermore, since $\mathbf{h}_{\mathbf{p}}$ is Gaussian, $\left|\mathbf{h}_{\mathbf{p}}\right|^{2}$ is a chi-square random variable with $2 M_{T} M_{R}$ degree of freedom and has the following moment generating function (MGF)

$$
\mathbb{E} \exp \left(-s\left|\mathbf{h}_{\mathbf{p}}\right|^{2}\right)=(1+s)^{-M_{T} M_{R}}
$$

Applying the MGF to (15), we obtain

$$
\begin{gathered}
\mathbb{M}(t ; \mathbf{X}) \\
=\mathbb{E}_{\tilde{\mathbf{h}_{\mathbf{p}}}}\left\{\mathbb{E}_{\left|\tilde{\mathbf{h}_{\mathbf{p}}}\right|^{2}} \exp \left[-\left(t \Omega\left|\tilde{\mathbf{h}_{\mathbf{p}}}\right|^{2}\right) \times\left|\mathbf{h}_{\mathbf{p}}\right|^{2}\right]\right\} \\
=\mathbb{E}_{\tilde{\mathbf{h}_{\mathbf{p}}}}\left\{\left((1+t \Omega)\left|\tilde{\mathbf{h}_{\mathbf{p}}}\right|^{2}\right)^{\left.-M_{T} M_{R}\right\}}\right.
\end{gathered}
$$

where the subscript on the expectation operator indicates the random variable being averaged over, and $\Omega$ is defined as

$$
\Omega \triangleq\left(\max _{\mathbf{x} \in \mathbf{X}}\left|\tilde{\mathbf{h}_{\mathbf{p}}}\right|^{2}\right)^{-M_{T} M_{R}} .
$$

Hence, we can be rewritten (16) as

$$
\begin{aligned}
\beta(\mathbf{X}) & =\lim _{t \rightarrow \infty} t^{M_{T} M_{R}} \mathbb{M}(t ; \mathbf{X}) \\
& =\lim _{t \rightarrow \infty} t^{M_{T} M_{R}} \mathbb{E}_{\tilde{\mathbf{h}_{\mathbf{p}}}}\left\{\left(1+\Omega^{\frac{-1}{M_{T} M_{R}}}\right)^{-M_{T} M_{R}}\right\} \\
& =\mathbb{E}_{\tilde{\mathbf{h}_{\mathbf{p}}}}(\Omega) .
\end{aligned}
$$

For $t>0$, it is to verify that $\left(1+t \Omega^{\frac{-1}{M_{T} M_{R}}}\right)-M_{T} M_{R}$ is concave with respect to $\Omega$. Thus, applying Jensen's inequality to (19), yielding:

$$
\begin{aligned}
\mathbb{M}(t ; \mathbf{X}) & \leq\left\{1+t\left[\mathbb{E}_{\tilde{\mathbf{h}_{\mathbf{p}}}} \Omega^{\frac{-1}{M_{T} M_{R}}}\right]^{-M_{T} M_{R}}\right\} \\
& =\left\{1+t\left[\beta(\mathbf{X})^{\frac{-1}{M_{T} M_{R}}}\right]^{-M_{T} M_{R}}\right\} .
\end{aligned}
$$


Substituting (22) into (14), an upper bound on the average BER is obtained

$\overline{\operatorname{BER}}(\rho) \leq \frac{1}{\pi} \int_{0}^{\frac{(M-1) \pi}{M}}\left(1+\frac{C \rho}{\sin ^{2}(\theta)} \beta(\mathbf{X})^{\frac{-1}{M_{T} M_{R}}}\right)^{-M_{T} M_{R}} d \theta$

\section{Simulation Results}

In this section, we present some simulation results to illustrate the performance of the proposed scheme. In order to assess the array gain of the proposed scheme, we also provide the performances obtained by other two closed-loop codebookbased schemes with four transmit antennas: the EO-STBC [10] and the ACBS [11].

In our simulations, we assume that the symbols are mapped to a QPSK constellation and we adopt as a stopping criterion the occurrence of 300 symbol errors per average SNR. We also assume the receiver knows the channel perfectly and sends $b$ bits over an error-free and zero-delay feedback channel. All the schemes considered here are rate one, i.e., for $M$-PSK constellations we have a transmission rate $R=\frac{Q}{\tau} \log _{2}(M)=$ $\log _{2}(M)$. The BER performance for the no-diversity scenario (single-input single-output scheme) is also plotted in Fig. 1, used as a reference curve.

The performance results are compared in terms of BER versus $\rho$ over quasi-static flat Rayleigh fading channels. In Fig. 1 and 2, results are given for $M_{T}=4$ transmit antennas and $M_{R}=1$ receive antenna. In Fig. 3, results are given for $M_{T}=4$ transmit antennas and $M_{R}=2$ receive antennas.

Fig. 1 shows the BER performance of the proposed, EOSTBC and ACBS schemes. For this simulations it was assumed an ideal (unquantized) feedback channel. We can observe that the proposed scheme achieves diversity order of $M_{T} M_{R}=4$ (the same order achieved by the other ones) and has a performance gain of about $2.4 \mathrm{~dB}$ over the EO-STBC and ACBS schemes.

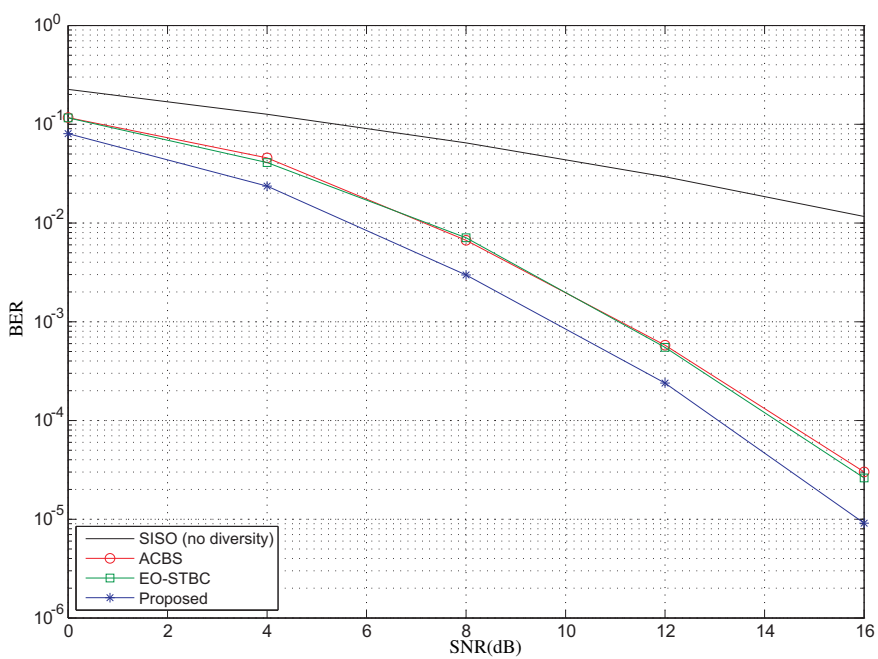

Fig. 1. BER performances. $M_{T}=4$ transmit antennas, $M_{R}=1$ receive antena, and $b=\infty$ feedback bits (unquantized).

$\mathbf{X}_{8}=\left[\begin{array}{cccc}\cos (\pi / 4) & \cos (\pi / 4) & \sin (\pi / 4) & \sin (\pi / 4) \\ \cos (\pi / 4) & \cos (\pi / 4) & \sin (\pi / 4) & \sin (\pi / 4) e^{j \pi} \\ \cos (\pi / 4) & \cos (\pi / 4) e^{j \pi} & \sin (\pi / 4) & \sin (\pi / 4) \\ \cos (\pi / 4) & \cos (\pi / 4) e^{j \pi} & \sin (\pi / 4) & \sin (\pi / 4) e^{j \pi} \\ \cos (-\pi / 4) & \cos (-\pi / 4) & \sin (-\pi / 4) & \sin (-\pi / 4) \\ \cos (-\pi / 4) & \cos (-\pi / 4) & \sin (-\pi / 4) & \sin (-\pi / 4) e^{j \pi} \\ \cos (-\pi / 4) & \cos (-\pi / 4) e^{j \pi} & \sin (-\pi / 4) & \sin (-\pi / 4) \\ \cos (-\pi / 4) & \cos (-\pi / 4) e^{j \pi} & \sin (-\pi / 4) & \sin (-\pi / 4) e^{j \pi}\end{array}\right]$

Fig. 2 and 3 show the BER performance of the proposed scheme for $M_{R}=1$ and $M_{R}=2$, respectively. For these simulations we have considered the codebooks $\left(\mathbf{X}_{8}\right)$ for $b=3$ and $\left(\mathbf{X}_{16}\right)$ for $b=4$ (see Table I for more details). Fig. 2 and 3 also present the theoretical upper bounds for $M_{R}=1$ and $M_{R}=2$, respectively. We note that the proposed and theoretical performances get closer as the number of feedback bits is increased.

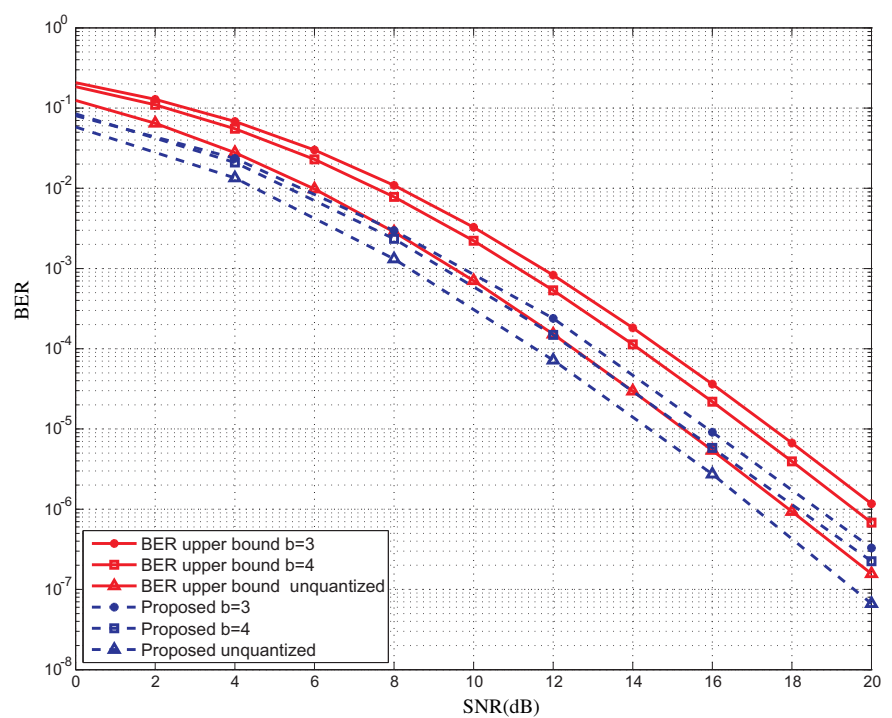

Fig. 2. BER performance: Theoretical upper bound and the performance of the proposed scheme for $M_{R}=1$. 


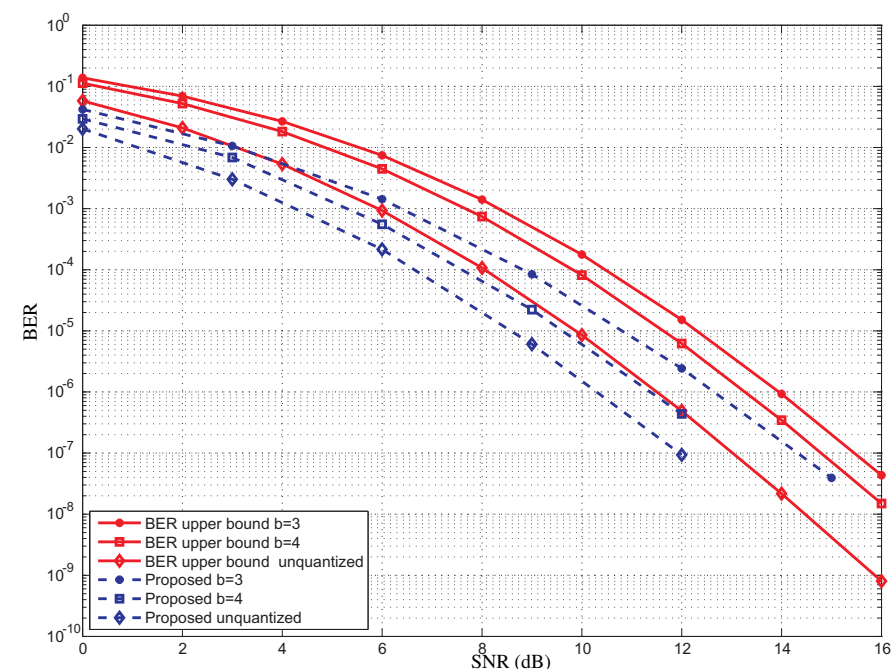

Fig. 3. BER performance: Theoretical upper bound and the performance of the proposed scheme for $M_{R}=2$.

\section{CONCLUSIONS AND FINAL REMARKS}

In this paper, a low-complexity codebook-based beamforming with four transmit antennas and quantized feedback channel was proposed. The proposed scheme is based on a preprocessing vector that combines the effect of phase rotation and power allocation (suboptimal in this proposal) into a new simple codebook-based transmission scheme.

The proposal can be seen as a code selector scheme, which allows us to evaluate it as a codebook-based design. An instantaneous SNR analysis was addressed in order to find the optimal feedback information. The proposed scheme was evaluated in terms of BER performance through Monte Carlo simulations. Further, a quantized feedback analysis over quasistatic flat Rayleigh fading channels was considered, and an upper bound on the BER performance was derived as well.

\section{ACKNOWLEDGEMENTS}

This work was partially supported by the Brazilian agency National Council for Scientific and Technological Development (CNPq) under grant 211271/2013-6, the SwedishBrazilian Research and Innovation Centre (CISB), Brazilian Army, and Saab AB.

\section{REFERENCES}

[1] M. Skoglund and G. Jongren, "On the capacity of multiple-antenna communication link with channel side information," IEEE Journal Sel. Areas Commun., vol. 21, no. 3 pp. 395-405, April 2003.

[2] P. Xia and G. B. Giannakis, "Design and analysis of transmitbeamforming based on limited-rate feedback," IEEE Trans. Signal Process., vol. 54, no. 5, pp. 1853-1863, May 2006.

[3] E. G. Larsson and P. Stoica, Space-Time Block Coding for Wireless Communications, Cambridge University Press, Cambridge, UK, 2003.

[4] D. Gore and A. Paulraj, "MIMO antenna subset selection with spacetime coding," IEEE Trans. on Signal Proc., vol. 50, no. 10, pp. 25802588, Dec. 2002.

[5] Z. Chen, J. Yuan, and B. Vucetic, "Analysis of transmit antenna selection/maximal-ratio combining in Rayleigh fading channels," IEEE Tran. Veh. Technol., vol. 54, no. 4, pp. 1312-1321, July 2005.

[6] K. K. Mukkavilli, A. Sabharwal, E. Erkip, and B. Aazhang, "On beamforming with finite rate feedback in multiple-antenna systems," IEEE Trans. Inf. Theory, vol. 49, no. 10, pp. 2562-2579, Oct. 2003.

[7] D. J. Love and R. W. Heath Jr., "Grassmannian beamforming for multiple-input multiple-output wireless systems," IEEE Trans. Inf. Theory, vol. 49, no. 10 , pp. 2735-2747, Oct. 2003.

[8] C. R. Murthy and D. Rao, "Quantization methods for equal gain transmission with finite rate feedback," IEEE Trans. Signal Processing, vol. 55, no. 1, pp. 233-245, Jan. 2007.

[9] D. Shin, H. Cho, and K. Yang, "General closed-form expressions for the SER and BER of decision-feedback detection with error propagation over MIMO fading channels," IEEE Tran. Veh. Technol., vol. 61, no. 2, pp. 882-888, Feb. 2012.

[10] N. M. Eltayeb, S. Lambotharan, and J. A. Chambers, "A phase feedback based extended space-time block code for enhancement of diversity," in Proc. IEEE VTC'07, April 2007, pp. 2296-2299.

[11] I. Choi, Jong-Kyu Kim, H. Lee, and I. Lee, "Alamouti-codes based four-antenna transmission schemes with phase feedback," IEEE Communications Letters, vol. 13, no. 10, pp. 749-751, Oct. 2009.

[12] P. Zhu, L. Tang, Y. Wang, and X. You, "An upper bound on the SER of transmit beamforming in correlated Rayleigh fading," IEEE Trans. Commun., vol. 58, no. 2, pp. 457-462, Feb. 2010.

[13] http://www.3gpp.org/Future-Radio-in-3GPP-300-attend

[14] Shengli Zhou, Zhengdao Wang, Georgios B. Giannakis, "Quantifying the Power Loss When Transmit Beamforming Relies on Finite-Rate Feedback," IEEE Trans. Wireless Commun., vol. 4, no. 4, pp. 19481957, July 2005.

[15] M. K. Simon and M-Slim Alouini, Digital Communication over Generalized Fading Channels, John Wiley and Sons, 2nd ed., New Jersey, 2005. 